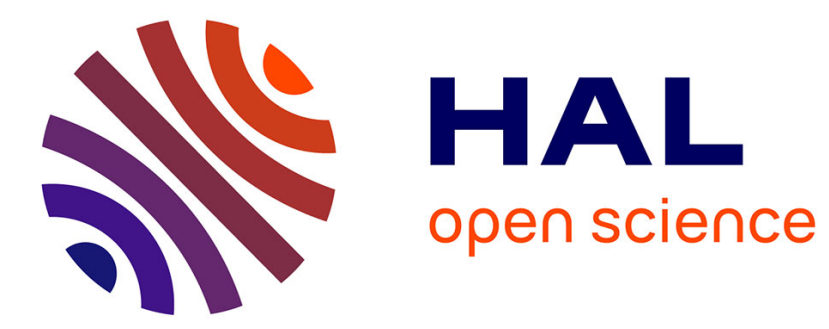

\title{
Misdirections in slow, goal-directed arm movements are not primarily visually based
}

J B de Graaf, A C Sittig, J J Denier van Der Gon

\section{To cite this version:}

J B de Graaf, A C Sittig, J J Denier van Der Gon. Misdirections in slow, goal-directed arm movements are not primarily visually based. Experimental Brain Research, 1994, 99, pp.464-472. hal-01453481

\section{HAL Id: hal-01453481 \\ https://hal.science/hal-01453481}

Submitted on 22 Nov 2017

HAL is a multi-disciplinary open access archive for the deposit and dissemination of scientific research documents, whether they are published or not. The documents may come from teaching and research institutions in France or abroad, or from public or private research centers.
L'archive ouverte pluridisciplinaire HAL, est destinée au dépôt et à la diffusion de documents scientifiques de niveau recherche, publiés ou non, émanant des établissements d'enseignement et de recherche français ou étrangers, des laboratoires publics ou privés. 
J. B. de Graaf - A. C. Sittig

J. J. Denier van der Gon

\section{Misdirections in slow, goal-directed arm movements are not primarily visually based}

Received: 7 January 1993 / Accepted: 14 October 1993

\begin{abstract}
In a previous study we found that the initial direction of slow, goal-directed arm movements deviates consistently from the direction of the actual straight line between the starting position and the target position. We now investigate whether these deviations are caused by imperfections or peculiarities in the processing of vision-related spatial information, such as retinal information, and eye- and head-position information. This could lead to incorrect localization of the target relative to the starting position. Subjects were seated in front of a horizontal surface and had to move their arm slowly and accurately in the direction of target positions. We varied the amount of vision-related spatial information. In experiment 1 , subjects were presented with visual targets and could see their moving arm. In experiment 2 , the subjects were again presented with visual targets, but now they could not see their moving arm. In experiment 3 , the subjects were blindfolded and had to move their arm towards tactile targets. In all three experiments we found comparable consistent deviations in the initial movement direction. We also instructed congenitally and early-blind subjects to move their arm towards tactile targets. Their performance showed deviations congruous with those found in the
\end{abstract}

J. B. de Graaf $^{1}$ - A. C. Sittig $(\square \nabla)$

Department of Product and Systems Ergonomics, Delft University of Technology, Jaffalaan 9,

NL-2628 BX Delft, The Netherlands

J. J. Denier van der Gon

Department of Medical and Physiological Physics,

University of Utrecht, Princetonplein 5,

NL-3584 CC Utrecht, The Netherlands

${ }^{1}$ Present address: Vision et motricité, INSERM Unité 94, 16 Avenue du Doyen Lépine, F-69500 Bron, France sighted subjects, and possibly somewhat larger. We conclude that the deviations in the initial movement direction of slow, goal-directed arm movements are not primarily visually based. The deviations are generated after all spatial information has been integrated.

Key words Spatial localization - Initial movement direction - Internal representation · Blindness · Human

\section{Introduction}

In order to execute a goal-directed movement we need to have a representation of our body and the space around us. We obtain this internal representation and update it constantly by using and combining information from various input channels, such as visual and proprioceptive systems and efference copies. In this study we investigate the internal representation of spatial relations that exists before the actual start of a goaldirected movement. Therefore, we pay special attention to the very first part of the movement trajectories. This stage of a movement best reflects the information available before the internal representation is updated during the movement.

In a previous paper (de Graaf et al. 1991b) we showed that, when human subjects are instructed to move their arm slowly and accurately in the direction of a visual target, they start moving their arm towards most target positions in a direction that consistently deviates from the target direction. Since they accurately reach the target position, the movement trajectories are curved. It should be emphasized that most subjects are convinced that they have moved their arm in a straight line towards the target position.

Since information about the origin of the deviations will give us insight into the process of spatial 
localization and into mechanisms generating control parameters of movements, we further investigate the origin of the deviations. An obvious cause might seem that the movement trajectories are influenced by biomechanical factors, such as inertia. However, this is unlikely for several reasons. Firstly, the movements were very slow $(3-5 \mathrm{~cm} / \mathrm{s})$, and it is unlikely that the mechanics of the effector system influence the trajectories of such slow movements. Secondly, several results show that the deviations also occur in comparable tasks, but where no arm movements are performed. In the same paper (de Graaf et al. 1991b), we showed that directing a pointer towards the same visual targets results in comparable deviations. In another paper we demonstrated misalignments in a visual, three-dot alignment task in a fronto-parallel plane that were very similar to the deviations in the pointer-setting task (Sittig and de Graaf 1994). Thus, from these results it appears that the consistent deviations in initial movement direction are not caused by mechanical properties of the effector system. The movements appear to be planned to start in a direction that deviates from the direction of the actual straight line between starting and target position. Furthermore, these results also make it unlikely that the deviations are planned to compensate for inertial and Coriolis forces that influence faster movements. Since the tasks in the above-mentioned experiments were performed mainly on the basis of visual spatial information (the targets were presented visually, and the subjects had visual feedback during the performance of the task), the results strongly suggest that the demonstrated deviations originate from imperfections or peculiarities in the processing of vision-related spatial information, such as retinal information, and eye- and head-position information. This could give rise to incorrect localization of the target position relative to the starting position. Such an idea of incorrect localization of relative positions has been proposed before (Hollins and Kelley 1988).

In the present study we investigated whether such visual mislocalization of relative starting and target position does indeed cause the deviations in initial movement direction. We did this by varying the amount of vision-related spatial information available to the subject during the performance of slow, goaldirected arm movements.

Three experiments were performed. In one experiment, the subjects were presented with visual targets and they could see their moving arm. Contrary to the instruction in the previously performed experiments (de Graaf et al. 1991b), the subjects were now free to move their head. In a second experiment, the subjects were again presented with visual targets, but now vision of their moving arm was prevented. This means that now proprioceptive information about hand position had to be combined with visual information about the target position. In a third experiment, the subjects were blindfolded and had to move their arm towards tactile targets, which were located by the index finger of the non-moving arm. This means that the subjects had to localize starting position as well as target position by proprioceptive information.

To investigate the influence of lifelong visual experience on the occurrence of the deviations, we also had congenitally blind subjects perform similar goaldirected arm movements. It is known that the interpretation of sensory information is influenced by signals from other sensory systems (e.g. Lackner 1973; Welch 1978). Since congenitally blind subjects have never learned to integrate proprioception with visual information, previous visual experience cannot possibly influence the execution of movements.

Preliminary results have been published in the form of an abstract (de Graaf et al. 1991a).

\section{Materials and methods}

Subjects

Nine normally sighted subjects (five men, four women; age 25-64 years) and five blind subjects (two men, three women; age 25-50 years) participated in this study. The normally sighted subjects and four of the blind subjects were right-handed, one blind subject was left-handed (subject B.G.). Two of the authors participated as subjects. Their results, however, were similar to those for the other sighted subjects.

The sighted subjects had normal or corrected-to-normal vision. Three of the blind subjects were congenitally blind due to retinitis pigmentosa (subject M.S.) or glaucoma (subjects B.G. and H.L.). Two blind subjects had lost their sight at a very early age ( 9 months, subject A.H., and 2 years, subject J.L.) due to retinoblastoma.

\section{Setup and procedure}

Three experiments were performed. The experiments differed only in the way the starting and target positions were presented and in the procedure followed. In all three experiments the subjects were seated in front of a horizontal table with their lower arm roughly horizontal. Movements of the shoulder, the elbow and the wrist were not restricted. On the table were seven (experiment 2) or nine (experiments 1 and 3) possible target positions and one starting position. The positions of the targets with respect to the starting position are depicted in Fig. 1A. The eyes of the subjects were approximately $45 \mathrm{~cm}$ above and $25 \mathrm{~cm}$ behind the starting position. The subjects were instructed to move the index finger of their dominant hand slowly and accurately from the starting position in the direction of the target position. They were asked to keep moving their finger accurately in the direction of the target and to stop the arm movement as soon as they reached, or thought they had reached, the target position (in experiment 2 they moved their finger only over a distance of approximately $15 \mathrm{~cm}$ ). The subjects were allowed to move their eyes and to orient their head towards starting and target positions. The way in which the targets and the starting position were presented to the subjects and the procedure varied across the experiments. 
A

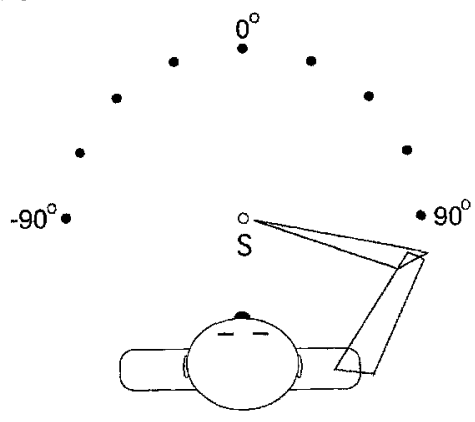

B

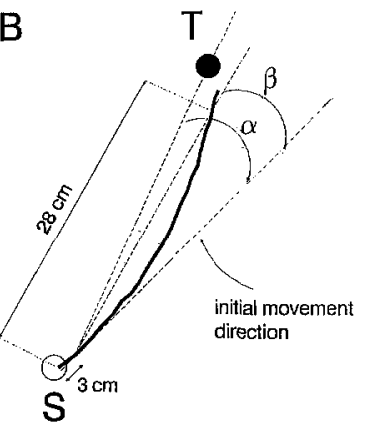

Fig. 1 A Stimulus surface seen from above. The targets are located on a semicircle with a radius of $31 \mathrm{~cm}$. The target position is indicated by the angle between the straight ahead and the line from the starting position $(S)$ to the target position. The target positions, in clockwise direction, are $-90^{\circ},-67.5^{\circ},-45^{\circ},-22.5^{\circ}, 0^{\circ}$, $22.5^{\circ}, 45^{\circ}, 67.5^{\circ}, 90^{\circ}$ (in experiment 2 the $\pm 90^{\circ}$ targets were not presented). B Explanation of the analysis. $T$ is a target position; $\alpha$ is

\section{Experiment 1: visual targets, moving arm visible}

Six sighted subjects participated. The nine targets and the starting position were indicated by light-emitting diodes (LEDs) placed in the stimulus surface. The subjects could see their moving arm and the surroundings were normally illuminated. The LEDs were only visible when lit. The presentation of the LEDs was computer controlled.

A trial took the following form. The subject placed the index finger on the starting LED. A target position was chosen quasi-randomly from the nine target LEDs. As soon as a target LED appeared, the starting LED went out. The target LED remained visible for $15 \mathrm{~s}$ during which time the subject moved the hand from the starting position to the target position. When the target LED went out, the starting LED appeared again and the subject placed the index finger on this LED.

To investigate how accurately subjects could move their arm in a straight line when no target position was presented, we held a few sessions in which subjects were asked to move their arm slowly and accurately in a straight line. Three subjects participated. Because we wanted to compare these trajectories with the trajectories of the goal-directed movements, the directions in which the subjects moved their arm had to be comparable in the two tasks. Therefore, nine LEDs were positioned at a distance of approximately $2 \mathrm{~cm}$ from the starting position to suggest initial movement directions. These LEDs remained visible only for a short time.

All target positions were presented five times. In some of the trials the subjects reported that they had started the movement in the wrong direction. These trials were not included in the analysis. It turned out that for a given target position not more than one trial had to be excluded. In the sessions where subjects were asked to move their arm in a straight line, the trials in which they reported that they had not moved their arm in a straight line were excluded.

\section{Experiment 2: visual targets, moving arm not visible}

Six sighted subjects participated. The seven targets and the starting position were indicated by LEDs and were only visible when they were lit. The presentation of the LEDs was computer controlled. In this experiment a shield was placed in between the head and arm of the subjects, such that they could see the targets but not their arm. The shield was such that it excluded vision of the whole arm, including the shoulder.

A trial took the following form. The subjects placed their index finger on the starting LED, then the shield was put in place. Next the deviation in initial movement direction; $\beta$ is the deviation with respect to the point the subjects reached after moving $28 \mathrm{~cm}$. If the trajectories are straight lines then $\beta=0$. A clockwise deviation is defined as positive. Note that positive deviations in the right half and negative deviations in the left half of the stimulus surface are deviations from target direction, away from the medial plane

a target was chosen quasi-randomly from the seven target LEDs. The target LED remained visible for $10 \mathrm{~s}$, during which time the subjects moved their hand approximately $15 \mathrm{~cm}$ in the direction of the target LED. They had to stop the movement at that distance so that they would not see their hand near the edge of the shield. When the target LED went out, the shield was removed and the subjects placed the index finger on the starting LED again.

All target positions were presented five times. The trials in which the subjects reported that they had not started their arm movement in the direction of the target were excluded. It turned out that, for a given target position, not more than one trial had to be excluded.

\section{Experiment 3: tactile targets, blind or blindfolded}

Six sighted and five blind subjects participated; the sighted subjects were blindfolded. The nine target positions and the starting position were indicated by small tactile dots. The target dots were placed on the underside of the stimulus table, the starting dot was placed on the table top. The stimulus table was $5 \mathrm{~mm}$ thick. For two of the blind subjects (H.L. and M.S.) only seven target positions were used, evenly distributed over the same semicircle.

The procedure in this experiment was as follows. The sighted subjects were not allowed to see the positions of the tactile dots on the underside of the stimulus table. All subjects first explored the underside of the table with their hands to get used to the positions of the nine tactile dots. At the beginning of each trial, the experimenter asked the subject to place the index finger of the non-moving arm on a specific, quasi-randomly chosen, target position. Next, the subject placed the index finger of the other arm on the starting position on the table and started the arm movement towards the target position. Thus, the subjects actively placed their hand on the tactile target as well as on the starting position.

As in experiment 1 , a few sessions were performed to investigate how accurately subjects could move their arm in a straight line when no target position was presented. Two blind subjects participated. No dots were used to indicate the initial movement direction. The subjects were asked to choose movement directions so that the whole range of directions between 90 and $-90^{\circ}$ would be covered.

All target positions were presented five times (for two of the blind subjects, H.L. and M.S., four times). The trials in which the subjects reported that they had started the movement in the wrong direction were excluded and repeated. In the sessions where subjects were asked to move their arm in a straight line, the trials in which they reported that they had not moved in a straight line were excluded and repeated. 


\section{Movement recording}

The movements were recorded with an Optotrak 2010 system. This system measures the three-dimensional (3D) coordinates of an infrared LED with an accuracy of $0.5 \mathrm{~mm}$ in all directions. This infrared LED was attached to the index finger of the moving arm. The position data were sampled at a frequency of $100 \mathrm{~Hz}$. The movement trajectories of two blind subjects (H.L. and M.S.) were measured in a different way: a small pencil was attached to the index finger of the moving arm in such a way that the subjects drew a line on a piece of paper when moving their finger.

\section{Analysis}

We calculated the initial direction of the movement as follows. Because in many trials the onset of the movements showed some irregularities (possibly due to friction), we did not use the position data for the first $2 \mathrm{~cm}$ of the movement to determine the initial movement direction. Instead, we used position data when the index finger was between 2 and $4 \mathrm{~cm}$ radial distance from the starting position. We determined the straight line through these data points by a least squares fit. The orientation of the resulting line is what we call the initial movement direction. The deviation in initial movement direction is the angle between this line and the line between the point on this line at a radial distance of $3 \mathrm{~cm}$ from the starting position and the target position ( $\alpha$ in Fig. 1B). (We could also have taken the line through the actual starting position and the target position. However, firstly, this does not seem to be the best choice: since the subjects were instructed to move in the direction of the target at every moment during the execution of the movement, the line through the point at $3 \mathrm{~cm}$ from the starting position and the target position is the direction in which they should move at that particular moment. Secondly, both ways of calculating the devi ations would produce the same pattern of deviations and would differ only slightly in the absolute value of the deviations. This would not alter our conclusions.) A clockwise deviation is a positive deviation in initial movement direction. Note that a clockwise deviation in the right half of the stimulus surface and an anticlockwise deviation in the left half of the stimulus surface are deviations from target direction, away from the medial plane. A deviation in initial movement direction could be determined within an accuracy of $2^{\circ}$ (measurement accuracy). For the two blind subjects whose trajectories were recorded with a pencil, the initial movement direction was determined by accurately fitting the best line on sight through the movement trajectories between 2 and $4 \mathrm{~cm}$ from the starting position.

To get an indication of the general course of the movement trajectories we determined the deviation in initial movement direction with respect to the point the subject's arm reached at a radial distance of $28 \mathrm{~cm}$ from the starting position ( $\beta$ in Fig. 1B). If the movement trajectories are straight lines these deviations will be zero. We used data at $28 \mathrm{~cm}$ from the starting position instead of data at the end position of the movements because the end position was not always at the same distance in these sessions. The value of $28 \mathrm{~cm}$ was taken because the subjects hardly ever stopped their arm movement before they had reached a distance of $28 \mathrm{~cm}$ from the starting position. Note that this distance almost equals the distance from starting position to target position $(31 \mathrm{~cm})$. The measurement accuracy of this "deviation" in initial movement direction is $2^{\circ}$. We used
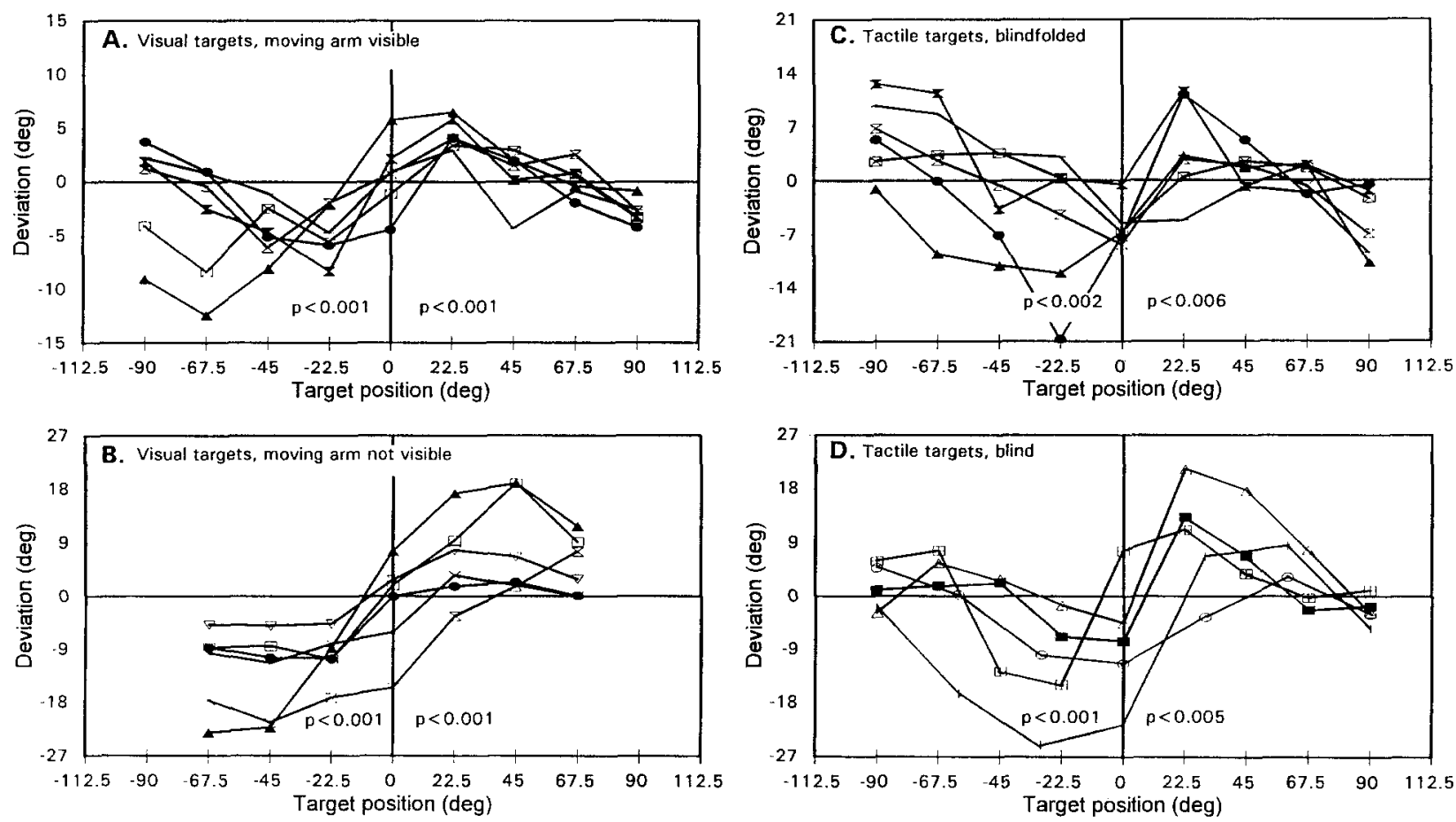

Fig. 2 Deviation in initial movement direction as a function of target position for the sighted (A-C) and five blind (D) subjects. Different symbols indicate different subjects. Each point represents the mean of four or five trials. The $P$ values are significance levels resulting from a one-tailed $t$-test, which was used for testing whether the initial movement direction of movements towards the $22.5^{\circ}$ and $-22.5^{\circ}$ target positions deviates significantly from target direction, away from

the medial plane. Note the scale differences. A Experiment 1: visual targets, moving arm visible. The mean standard deviation (SD) is $2.8^{\circ}$. B Experiment 2: visual targets, moving arm not visible. SD is $4.3^{\circ}$. C Experiment 3: tactile targets, blindfolded. SD is $4.8^{\circ}$. D Experiment 3: tactile targets, blind. SD is $6.7^{\circ}$. ( | M.S., $\bigcirc$ H.L., $\square$ B.G., $\square$ A.H., $\triangle$ J.L.) For two blind subjects (H.L. and M.S.) we used the movements towards the $30^{\circ}$ target and the $-30^{\circ}$ target for statistical testing 
this analysis of the general course of the trajectory in the sessions where the subjects were instructed to move their arm along a straight line and in experiment 3 (tactile targets, blind or blindfolded).

\section{Results}

This study was performed to investigate whether visual mislocalization could be responsible for the misdirections of slow, goal-directed arm movements. We were interested in the initial movement direction and the general course of the movements.

Experiment 1: visual targets, moving arm visible

We determined the deviation in initial movement direction. Figure 2 shows mean results for each subject in all three experiments. The deviation in initial movement direction is presented as a function of target position. Figure 2A demonstrates that when subjects move their arm towards visual targets, the deviations in initial movement direction are positive for movements towards targets in the right half of the stimulus table, and negative for movements towards targets in the left half of the stimulus table. These results are comparable with the results of the previous study (de Graaf et al. 1991b). In none of the experiments did the standard deviation significantly depend on target position or subject. We therefore calculated the mean standard deviation in initial movement direction using the standard deviations for movements towards all target positions and for all subjects. In experiment 1 the mean standard deviation was $2.8^{\circ}$.

In the previous study we found clear deviations in initial movement direction for target positions of roughly $22.5^{\circ}$ and $-22.5^{\circ}$. Therefore, in the present study we used the results for these target positions for statistical testing. A $t$-test for comparison of sample means was used to determine whether the deviations in the initial movement direction of movements to the $22.5^{\circ}$ target were significantly positive and to the $-22.5^{\circ}$ target, significantly negative. If they are, then we state in the following that movements in the right half and in the left half of the stimulus table show consistent deviations from target direction, away from the medial plane. The $P$-values are given in Fig. 2 and 3. In experiment 1 the initial movement direction for movements in the right half as well as in the left half of the stimulus surface was indeed found to deviate significantly from target direction away from the medial plane.

A few sessions were performed in which subjects were asked to move their arm in a straight line instead of in the direction of a target position. Figure 3 shows the "deviations" in initial movement direction (see Materials and methods) as a function of the initial
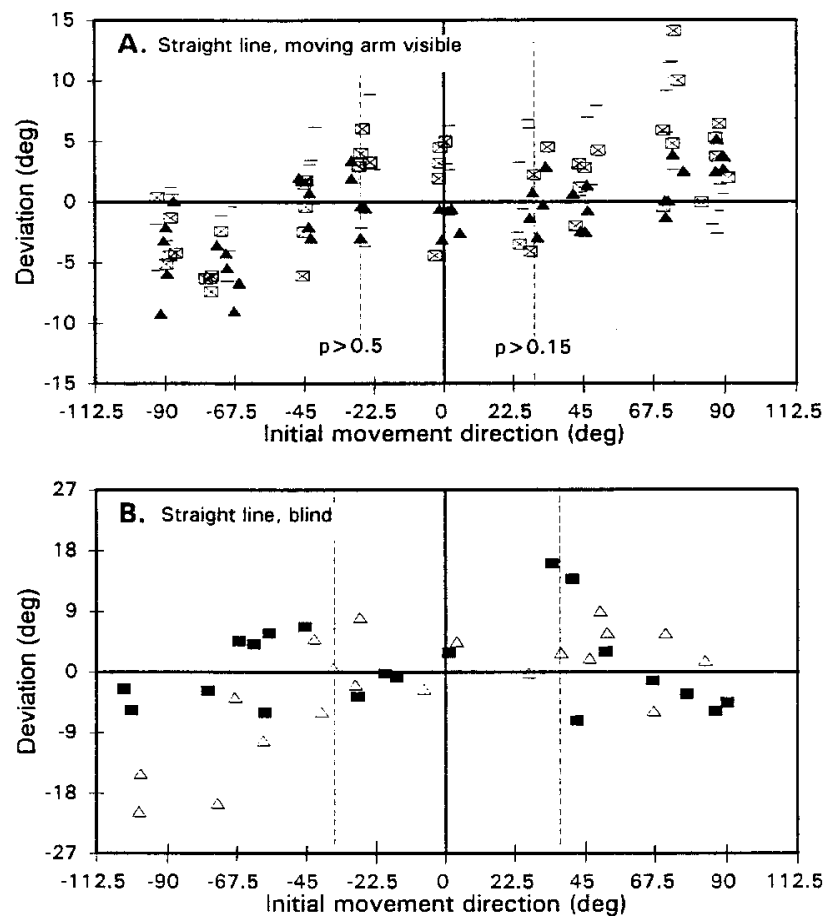

Fig. 3A, B Deviation ( $\beta$ in Fig. 1B) as a function of initial movement direction. Subjects had been instructed to move their arm in a straight line. Each point is a single trial. For explanation of $P$-values see Fig. 2. A Sighted subjects. Symbols indicate same subjects as in Fig. 2 . The $P$ values show that the deviations are not significantly away from the medial plane. B Blind subjects. Symbols indicate same subjects as in Fig. 2D. Vertical dashed lines indicate initial movement directions where we would expect significant deviations away from the medial plane. Note the small deviations for these directions

movement direction. Figure $3 \mathrm{~A}$ shows single trials for three sighted subjects. It can be seen that, except for initial movement directions of over $\pm 70^{\circ}$, the deviations are roughly scattered around zero. If the instruction to move the arm in a straight line had resulted in trajectories comparable with those in experiment 1, we would have expected significant deviations away from the medial plane for an initial movement direction of $27^{\circ}$ and $-27^{\circ}$ (which are roughly the initial movement directions of movements towards the targets $22.5^{\circ}$ and $-22.5^{\circ}$, respectively). As can be seen in Fig. 3A, however, the deviations for movements starting in a direction of $27^{\circ}$ and $-27^{\circ}$ were not significantly away from the medial plane. A $t$-test was used to compare the results of the different experiments. The $P$ values are shown in Table 1 . It can be seen that the deviations were significantly different for movements in a straight line and movements in the direction of a target. The deviations that we see for initial movement directions of over $\pm 70^{\circ}$ are most probably caused by restraints in the shoulder joint: it is difficult for the subjects to keep moving their arm in a straight line when their upper arm is in an extreme 
Table 1 Results of a two-tailed $t$-test to test whether the deviations in initial movement direction in the three experiments are significantly different (comparison of sample means). The deviations in initial movement direction for movements towards the $22.5^{\circ}$ and $-22.5^{\circ}$ target positions in the experiments were compared with the deviations of movements towards the same targets in the other two experiments. ( $b l$ blind subjects, $b l f$ blindfolded subjects, straight the sessions in which three sighted subjects had been instructed to move their arm in a straight line)

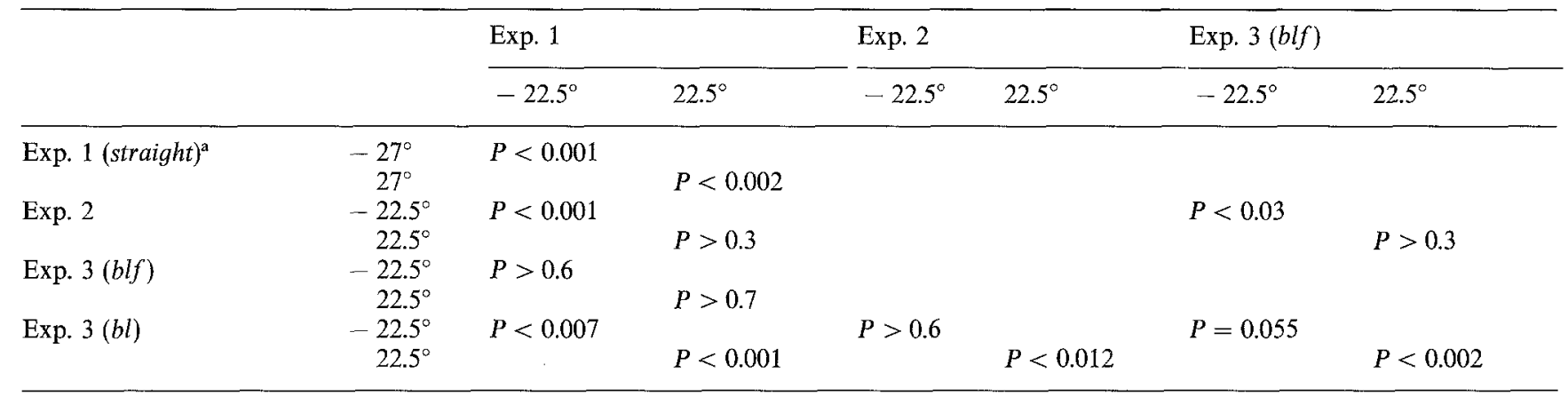

${ }^{a}$ Because we had no targets in these sessions, we used the results of movements with an initial movement direction of about $27^{\circ}$ and $-27^{\circ}$ (which are roughly the initial movement directions of the movements towards the $22.5^{\circ}$ and $-22.5^{\circ}$ target positions in experiment 1 ) position. We think that this does not affect the movements towards a target, because then this effect is overruled by the homing-in mechanism.

Experiment 2: visual targets, moving arm not visible

Figure 2B shows results for experiment 2 . It is clear that the subjects start their arm movements with similar deviations in the initial direction. Statistical testing reveals that the initial movement directions do indeed deviate significantly from target direction, away from the medial plane for left and right sides of the stimulus table. The mean standard deviation in this experiment is $4.3^{\circ}$. This is, as to be expected in a condition without visual feedback, somewhat larger than we found in experiment 1 .

The deviations appear to be somewhat larger than in experiment 1 (note scale difference). This is clearest for the left half of the stimulus table. Indeed, in Table 1 it can be seen that for the left half of the stimulus table the deviations are significantly larger than we found in experiment 1 . We did not find a significant difference for the right half of the stimulus table.

\section{Experiment 3: tactile targets, blind or blindfolded}

Figure $2 \mathrm{C}$ shows deviations in initial movement direction when blindfolded subjects move their arm to tactile targets. It can be seen that for five out of six subjects the deviations in initial movement direction roughly resemble the deviations we found in experiment 1 and 2 (Fig. 2A, B). Indeed, when subjects are blindfolded they start their arm movements to targets in the right half of the stimulus table as well as to targets in the left half of the stimulus table with a consistent deviation, away from the medial plane. The mean standard deviation of the deviations in initial movement direction is $4.8^{\circ}$.

In Table 1 it can be seen that the deviations in initial movement direction do not differ significantly from the deviations found in experiment 1 and for the right side of the stimulus table in experiment 2. However, for the left half of the stimulus table, the deviations are significantly smaller than we found in experiment 2 .

One might wonder here whether the curvatures of the trajectories were similar to those in experiment 1 (in experiment 2 the subjects stopped moving their arm at a distance of $15 \mathrm{~cm}$ from the target position). In other words, did the subjects end their movements on or near the target position? Looking at the movement trajectories, we saw that, although the variability in end position was clearly larger than in experiment 1 , the subjects did indeed end their movements on average near the target position. This is supported by the fact that the deviations in initial movement direction with respect to the point the subjects reached after moving their arm over $28 \mathrm{~cm}$ (see Materials and methods) do not differ significantly from the deviation in initial movement direction with respect to the target position $\left(P>0.1\right.$ for the $-22.5^{\circ}$ target and $P>0.6$ for the $22.5^{\circ}$ target).

These results clearly demonstrate that the initial movement direction and the general course of the movements are not essentially affected by whether or not the subjects can see the starting and the target position. To confirm this finding we had congenitally and early-blind subjects move their arm in the direction of tactile targets. Figure 2D shows deviations in initial movement direction for five blind subjects. Firstly, it can be seen that these subjects show consistent deviations in initial movement direction. The initial movement direction deviates significantly from target 
direction away from the medial plane. The mean standard deviation is $6.7^{\circ}$. Secondly, when the results of the blind subjects are compared with those of the blindfolded sighted subjects, the absolute value of the deviations in initial movement direction turns out to be larger for the blind subjects, both for movements in the right half of the stimulus surface (highly significant) and for movements in the left half of the stimulus surface (almost significant, see Table 1).

Also with regard to these subjects one might wonder about the general course of the movements. Did the subjects end their arm movements near the target position? Here too it appeared that on average they did end their arm movements on the target, although we found a large variability in end position. Again, we calculated the deviations in initial movement direction with respect to the point the subjects reached after moving $28 \mathrm{~cm}$. Statistical testing revealed no significant difference between this "deviation" and the deviation with respect to the target position for the $-22.5^{\circ}$ target $(P>0.7)$ and a hardly significant difference for the $22.5^{\circ}$ target $(P=0.048)$. This demonstrates that, although the subjects started their movements in a direction significantly deviating from the target direction, they ended their movements near the target position.

As in experiment 1 , a few sessions were performed in which blind subjects were asked to move their arm in a straight line. Figure 3B shows single trials for the two blind subjects. Some movements in the right half of the stimulus surface show positive deviations, but the values are on average much smaller here than in the trials where the subjects had been instructed to move their arm towards a target. Because it turned out that the subjects did not very often select an initial movement direction of $30^{\circ}$ and $-30^{\circ}$ (which were, for the blind subjects, roughly the initial movement directions of movements towards the $22.5^{\circ}$ and $-22.5^{\circ}$ targets, respectively) we did not compare these results statistically with the results of the sessions in which these subjects moved in the direction of a target position. We believe the results are clear enough to reach the conclusion that when blind subjects are instructed to make an arm movement in a straight line their movement trajectories are straighter than when they are asked to move their arm towards a target.

\section{Discussion}

This study investigated whether imperfections or peculiarities in the processing of vision-related spatial information, such as retinal information, and eye- and head-position information, could be responsible for the consistent deviations that we found in the initial movement directions when subjects move their arm slowly and accurately in what they think is the direction of visual targets.

The results of experiment 1 (visual targets, moving arm visible) closely resemble the results found previously (de Graaf et al. 1991b). When subjects move their arm towards visual targets, the initial movement direction deviates from target direction, away from the medial plane. In the previous study the subjects were not allowed to move their head while performing the task. In the present study, however, head movements were free. We saw them make substantial head movements, especially in trials with the more peripheral targets. So, contrary to the previous study, in the present study no extreme eye positions in the orbit occurred when subjects looked at the target position. The fact that the deviations are similar indicates that imperfections in the processing of eye position information resulting from not orienting the head towards the target (as is suggested by Biguer et al. 1984) is not an explanation for the deviations in initial movement direction. Also other explanations based on head movements, such as the idea of, e.g. Marteniuk (1978) and Roll et al. (1986) that head position cues contribute to the directional specification of aiming movements, do not seem to be a valid explanation for the deviations in initial movement direction. The deviations are not related to head orientation.

We suggested earlier (Sittig and de Graaf 1994) that the deviations in initial movement direction are related to the deviations in initial direction of saccades. The curvature of oblique saccades is somewhat similar to the curvatures we find in slow goal-directed arm movements (see, e.g. Smit and van Gisbergen 1990) and Viviani et al. (1977) for trajectories of oblique saccades). A speculative hypothesis is that the brain uses the initial direction of the saccade from starting to target position as an indication for the initial movement direction of the arm movement (Sittig and de Graaf 1994). Subjects did indeed make saccades in between starting and target position when performing the slow arm movements in our study (unpublished data). It was partly to test this hypothesis that we did experiment 2 .

In experiment 2 we excluded vision of the moving arm and thus of the starting position. The results show that the deviations in initial movement direction are similar to, or even somewhat larger than, the deviations when subjects can see their moving arm. We did not record eye movements, but careful observation revealed that in this experiment the subjects made no saccades between starting and target position. This is not surprising, since they could not see the starting position. (One might think that the subjects would still make eye movements towards the "felt" hand position, but they hardly ever did: they fixated the target position during the arm movements). Thus, it seems that the initial direction of the arm movement is not related to 
the actual saccades that are made towards the target position. Moreover, the initial direction of the movements does not seem to be essentially influenced by whether or not the subjects can visually relate starting and target position. When they have to relate proprioceptive information about the starting position with visual information about the target position, similar deviations in initial movement occur.

In experiment 3 we excluded vision of the starting position as well as of the target position. The results of this experiment, in which blindfolded sighted subjects and blind subjects moved their arm towards tactile targets, show that whether or not one has visual information of the target again does not essentially affect the initial movement direction. Even subjects who never had any visual information show consistent deviations in initial movement direction. These deviations are congruous with the deviations of (blindfolded) sighted subjects, but somewhat larger. This strongly suggests that the deviations in initial movement direction are certainly not caused by imperfections in the processing of vision-related spatial information. Visual experience might reduce the deviations, even if there is no visual information during the actual performance of the movements.

One might object that it is difficult for blind subjects to move their arm straight in the direction of a target position, since it seems to be a very "visual" instruction. It is possible that they simply moved their arm along a trajectory which was the easiest one for them. However, there are several reasons for believing that this was not the case. Firstly, when subjects were asked to move their arm in a straight line, blind subjects were able to do this. Thus, congenitally blind subjects do know what a straight line is. This is confirmed by studies in which blind subjects were given edges with various curvatures (Davidson 1972; Hunter 1954). They were able to say which edge was the straightest. In these studies the blind subjects in fact performed better than blindfolded sighted subjects. Secondly, most blind subjects immediately interpreted the instruction to move their arm in the direction of a target as an instruction to move in a straight line, which, as they said afterwards, is what they thought they had done. Thirdly, it is not at all obvious why moving their arm along the trajectories in the way they did would be easier than moving their arm along a straight line towards the target position.

Thus, our results strongly suggest that the deviations in initial movement direction are not visually based, i.e. the deviations are not caused by imperfections or peculiarities in the processing of vision-related spatial information. Then what is it that causes the deviations? In the literature one can find a number of theories about errors in manual pointing tasks. Although in these theories the actual execution of the movement is essential, and we showed that the devi- ations in initial movement direction are not caused by the motor component of the task (pointer-setting task, de Graaf et al. 1991b), the underlying processes might nevertheless be related. Soechting and Flanders $(1989 a, b)$ propose a model for the errors in end position which occur in pointing movements to remembered visual targets in 3D space. Their model is based on the notion that the spatial location of a visual target and the orientation of the arm are represented in different coordinate systems. The errors would result from approximations in the transformation of visual information about target location to a frame of reference used to represent the location of the hand in space. Although this model only accounts for errors in the end position, a similar process might hold for the initial movement direction. However, since we have shown that the deviations in initial movement direction are also present in a purely proprioceptive task (experiment 3: tactile targets, blind or blindfolded), the deviations cannot be caused by inaccuracies in the transformation from a visual frame of reference to a different one.

In their study with deafferented patients, Ghez et al. (1990) showed that proprioceptive information from the moving limb is necessary to compensate for mechanical anisotropy. Might the deviations in initial movement direction be caused by a failure to completely compensate for such an anisotropy? This is unlikely for several reasons. Firstly, since the movements of our subjects were very slow, the acceleration was low. This greatly reduces the influence of inertial forces. Secondly, the pattern of deviations in initial movement direction is not in agreement with the deviations resulting from mechanical anisotropy of the arm. For instance, one would not expect a symmetry of deviations around the subject's "straight ahead".

Hollins and Kelley (1988) propose a model that could explain misdirections and that does not rely on the execution of a movement. In their study blind and blindfolded sighted subjects were instructed to direct a pointer towards several remembered objects, the position of which they had learned by locating them by touch. They found deviations in pointer direction similar to the deviations in pointer direction that we found before (de Graaf et al. 1991b). They suggest that objects (targets) in space that are located far from the body are internally represented to be relatively closer to the body than objects that are actually located close to the body (a contraction towards the frontal plane and parallel to the medial plane of the subject). Planning a movement towards a target position that is located further from the body than the starting position (as was the case in our study) would then indeed result in deviations in initial movement direction such as those we found.

If we want to use the model of Hollins and Kelley (1988) to explain the deviations in initial movement direction that we found, it will have to be extended. 
Firstly, in their model the actual pointer position (and thus hand position) was not exposed to a contraction. But then the arm could never end on the target position, whereas usually, of course, arm movements do end on target positions (even when subjects are blind or blindfolded). If we extend the contraction model to all locations in space, then the hand is exposed to the same "distortion" and, consequently, all movements will end on the target position. The general idea of such a model would be that the actual movement trajectory corresponds to a straight line in the internal representation. Secondly, Hollins and Kelley (1988) did not state explicitly how the contraction of locations is related to distance from the frontal plane of the subject. A linear contraction of locations (i.e. the contraction is proportional to the distance from the frontal plane) would not predict deviations in initial movement direction. This is easy to see: a point in space that is located on the straight line through starting and target position would in the internal representation be located again on a straight line through the (contracted) starting and target position. Therefore, in order to find the deviations in initial movement direction that we found, the transformation between locations in the "real world" and the internal representation needs to be non-linear, i.e. the contraction of locations must be non-linearly dependent on the distance from the frontal plane of the subject. Then straight lines in the internal representation will be transformed into a movement trajectory that is actually curved.

One could argue that this model contradicts the finding in the present study that subjects can move their arm in a straight line if they are instructed to do so. Apparently, the internal representation of a straight line is transformed to an actual straight line in the "real world". However, the process of moving in a straight line is probably different from the process of moving towards a target. The task of moving an arm in a straight line might be performed by "not changing the movement direction", whereas moving an arm towards a target is probably more a process of "moving at every moment in the direction of the target position". Thus, this finding does not necessarily contradict the proposed model.

It is interesting to note that the construction of the internal representation of spatial relations is apparently not dominated mainly by visual information, as is often suggested. In the present study we show that congenitally blind subjects perform slow, goal-directed arm movements in very much the same way as sighted subjects. This suggests that the blind subjects have roughly the same internal representation of locations as the sighted subjects. However, blind subjects build their internal representation mainly on the basis of proprioceptive information, whereas the sighted subjects have access to more accurate information, namely vision. The fact that visual experience diminishes the deviations in initial movement direction only slightly may indicate that in sighted subjects proprioceptive information contributes strongly to the construction of the internal representation of spatial relations.

Summarizing, we have shown that the deviations in initial movement direction do not originate from imperfect processing of vision-related spatial information. The results suggest that the deviations are caused by a distorted internal representation of spatial relations. In a follow-up paper we will test this hypothesis by varying the location of the starting and target position with respect to the body of the subject.

Acknowledgement The authors wish to thank Sheila McNab for improving the language and clarity of the paper.

\section{References}

Biguer B, Prablanc C, Jeannerod M (1984) The contribution of coordinated eye and head movements in hand pointing accuracy. Exp Brain Res 55: 462-469

Davidson PW (1972) Haptic judgments of curvature by blind and sighted humans. J Exp Psychol 93: 43-55

Ghez C, Gordon J, Chilardi MF, Christakos CN, Cooper SE (1990) Roles of proprioceptive input in the programming of arm trajectories. Cold Spring Harb Symp Quant Biol 55: $837-847$

Graaf JB de, Denier van der Gon JJ, Sittig AC (1991a) Directional information during slow goal-directed arm movements. Soc Neurosci Abstr 17(2): 1110

Graaf JB de, Sittig AC, Denier van der Gon JJ (1991b) Misdirections in slow goal-directed arm movements and pointer-setting tasks. Exp Brain Res 84: 434438

Hollins M, Kelley EK (1988) Spatial updating in blind and sighted people. Percept Psychophys 43: 380-388

Hunter IML (1954) Tactile-kinaesthetic perception of straightness in blind and sighted humans. Q J Exp Psychol 6: 149-154

Lackner JR (1973) Visual rearrangement affects auditory localization. Neuropsychologia 11: 29-32

Marteniuk RG (1978) The role of eye and head positions in slow movement execution. In: Stelmach GE (ed) Information processing in motor control and learning. Academic, New York, pp 267-288

Roll R, Bard C, Paillard J (1986) Head orienting contributes to the directional accuracy of aiming at distant targets. Hum Movement Sci 5: 359-371

Sittig AC, Graaf JB de (1994) Orientation dependent misalignments in a visual alignments task. (In press)

Smit AC, Gisbergen JAM van (1990) An analysis of curvature in fast and slow human saccades. Exp Brain Res 81: 335-345

Soechting JF, Flanders M (1989a) Errors in pointing are due to approximations in sensorimotor transformations. J Neurophysiol 62: 595-608

Soechting JE, Flanders M (1989b) Sensorimotor representation for pointing to targets in three-dimensional space. J Neurophysiol 62: $582-594$

Viviani P, Berthoz A, Tracey D (1977) The curvature of oblique saccades. Vision Res 17: 661-664

Welch RB (1978) Perceptual modification: adaptation to altered sensory environments. Academic, New York 